\title{
INVESTIGATION OF VERTICAL SLAMMING ON POINT ABSORBERS
}

\author{
Griet De Backer \\ Ghent University \\ Coastal Engineering Division \\ Technologiepark Zwijnaarde 904 \\ B-9052 Zwijnaarde, Belgium \\ Griet.DeBacker@ugent.be
}

\author{
Marc Vantorre \\ Ghent University \\ Maritime Technology Division \\ Technologiepark Zwijnaarde 904 \\ B-9052 Zwijnaarde, Belgium \\ Marc.Vantorre@ugent.be
}

\author{
Sam Victor \\ Ghent University \\ Coastal Engineering Division \\ Technologiepark Zwijnaarde 904 \\ B-9052 Zwijnaarde, Belgium \\ Sam.Victor@ugent.be
}

\author{
Julien De Rouck \\ Ghent University \\ Coastal Engineering Division \\ Technologiepark Zwijnaarde 904 \\ B-9052 Zwijnaarde, Belgium \\ Julien.DeRouck@ugent.be
}

\author{
Charlotte Beels \\ Ghent University \\ Coastal Engineering Division \\ Technologiepark Zwijnaarde 904 \\ B-9052 Zwijnaarde, Belgium \\ Charlotte.Beels@ugent.be
}

\begin{abstract}
This paper focuses on the impact of vertical slamming on floating point absorber systems and the associated pressures that might be expected when these phenomena occur. In a first part it will be shown how the occurrence probability of slamming can be reduced by implementing a slamming restriction, i.e. by controlling the motion of the point absorber. The impact of these slamming restrictions on power absorption will be discussed. Secondly an investigation is made of the pressures that occur when the buoys are subject to vertical bottom slamming. Analytical results are presented, which give a pressure prediction of an impacting body with conical and hemispherical shape, using Wagner theory. Laboratory experiments have been carried out at Ghent University. Impact pressures were measured during drop tests with both hemispherical and conical buoy shapes. These pressures were measured by ICP pressure sensors with a range up to $345 \mathrm{kPa}$ with small membrane and very high resonance frequency $(>250$ $\mathrm{kHz}$ ). Analytical and physical results are compared and conclusions are drawn.
\end{abstract}

\section{KEY WORDS}

Point absorber; slamming; Wagner theory; ocean energy; drop tests

\section{INTRODUCTION}

Point absorbers are wave energy converters consisting of floating bodies oscillating with one or more degrees of freedom of which the horizontal dimensions are small in comparison to the wave length. Several Wave Energy Converters (WECs), composed of one or multiple point absorbers, are currently under development. Examples are $\mathrm{FO}^{3}$ [1], Wave Star [2], Manchester Bobber [3] and many other devices. At present intensive research is being carried out on shape optimization of point absorbers from the point of view of power absorption [4$6]$. However, the design of the buoy shape does not only influence the energy extraction but also the pressures and forces acting on the body. Oscillating point absorbers may loose contact with the water surface and be exposed to important impact pressures when re-entering the water. This phenomenon is called bottom slamming and it occurs particularly when the buoys have a small draft in combination with energetic wave conditions.

\section{NOMENCLATURE}

$b=$ instantaneous radius at the intersection point between the body and the water [m]

$b_{0}=$ instantaneous radius at $z=0[\mathrm{~m}]$

$B=$ hydrodynamic damping coefficient $[\mathrm{kg} / \mathrm{s}]$

$B_{\text {ext }} \quad=\quad$ external damping coefficient $[\mathrm{kg} / \mathrm{s}]$ 


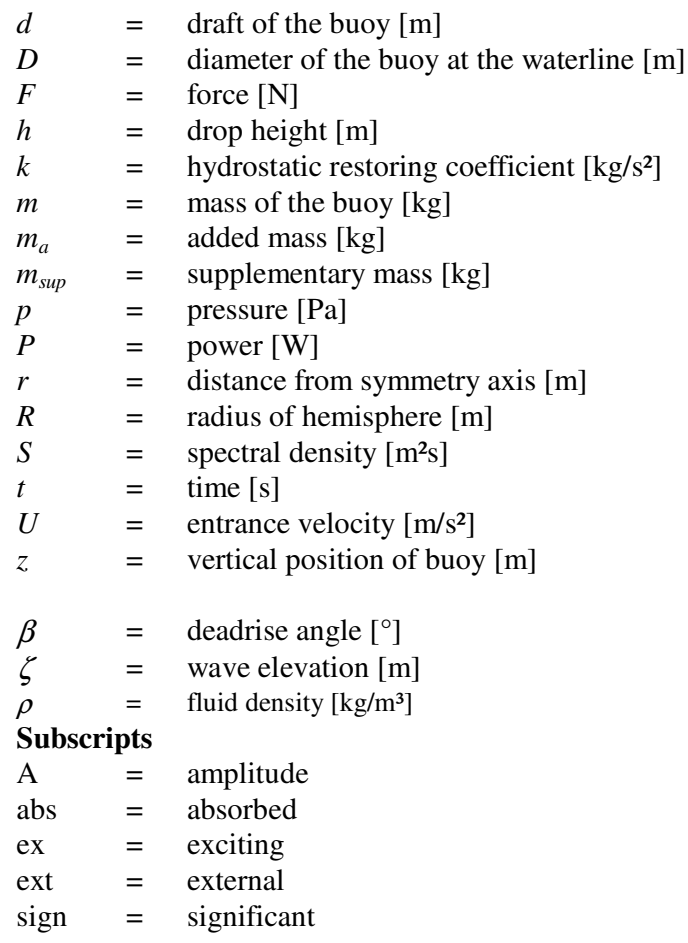

\section{INFLUENCE ON POWER ABSORPTION}

Before investigating the impact of slamming restrictions on power absorption, a brief introduction is given on how the power absorption is calculated, how the slamming restriction is formulated and fulfilled. With linear theory, the equation of motion of a heaving point absorber can be expressed as follows:

$$
\left(m+m_{\text {sup }}+m_{a}\right) \frac{d^{2} z}{d t^{2}}+\left(B+B_{e x t}\right) \frac{d z}{d t}+k z=F_{e x}
$$

with $z$ the position of the buoy and $m$ the mass of the buoy. The added mass of the buoy is denoted by $m_{a}, B$ is the hydrodynamic damping coefficient and $F_{e x}$ is the exciting wave force. These parameters depend on both the frequency and shape of the buoy. The hydrodynamic coefficients $m_{a}, B$ and $F_{e x}$ are calculated with a Boundary Element Method (BEM) package. Parameter $k$ is the hydrostatic restoring coefficient and is dependent on the buoy shape. The linear external damping coefficient, $B_{e x t}$, enables power absorption. The supplementary mass, $m_{\text {sup }}$, increases the natural period of the buoy. As such, this supplementary mass allows for an easy way of tuning the buoy to the incoming waves in a frequency domain model [4]. The forces associated with $B_{\text {ext }}$ and $m_{\text {sup }}$ have to be realised by the power take-off (PTO) and motion control system respectively.

The parameters supplementary mass and external damping coefficient are optimised in order to maximize the power absorption $P$, which can be expressed by:

$$
P=\int_{0}^{\infty} b_{e x t} \omega^{2}\left(\frac{z_{A}}{\zeta_{A}}\right)^{2} S_{\zeta}(\omega) d \omega
$$

with $\omega$ the pulsation, $z_{A}$ the amplitude of the buoy motion, $\zeta_{A}$ the wave amplitude and $S_{5}$ the wave spectrum. The amount of absorbed power is influenced by the external damping coefficient, $B_{e x t}$, and by the velocity of the buoy which is dependent on both $B_{\text {ext }}$ and the supplementary mass, $m_{\text {sup }}$.

In order to reduce the probability of slamming and to decrease the effects of slamming (by decreasing the entry velocity) a motion restriction is implemented. Slamming occurs when:

$$
(z-\varsigma)_{A}>d
$$

with $d$ being the draft of the buoy. To reduce the probability of slamming, the significant amplitude of the position of the buoy relative to the free surface is not allowed to be higher than the draft of the buoy: $(z-\varsigma)_{A, s i g n}<d$. This means that slamming is still allowed for the $13.5 \%$ highest waves. In small and moderate waves, the slamming criterion does not influence the optimal values of the control parameters. However, for higher waves and smaller buoy drafts, less optimal values of the control parameters $B_{\text {ext }}$ and $m_{\text {sup }}$ have to be chosen in order to fulfill the slamming criterion. Fig. 1 shows the absorbed power as a function of the supplementary mass and external damping coefficient for $H_{s}=2.25 \mathrm{~m}$ and $T_{z}=5.75 \mathrm{~s}$, for a heaving buoy with waterline diameter $\mathrm{D}=4 \mathrm{~m}$. The submerged part of the buoy consists of a cone shape with top angle $90^{\circ}$ and a cylindrical upper part of $0.5 \mathrm{~m}$. The total draft is $2.5 \mathrm{~m}$. The shaded area has to be avoided to fulfill the slamming restriction, resulting in less power absorption. As can be seen in the graph, the slamming constraint is satisfied when the point absorber is detuned (by selecting a less optimal supplementary mass), when the external damping is increased or by using a combination of both. With the help of a simple algorithm, the optimal combination of control parameters is selected in the remaining (non-shaded) area on the graph, resulting in maximum allowable power absorption, without violating the slamming restriction. The maximum power absorption in this remaining area is $45 \mathrm{~kW}$, which is indicated by a circle on the graph. This figure does not take into account losses due to mechanical friction, turbulent losses, turbine and generator losses or any other losses in the conversion system and is for that reason not equal to the produced electrical power.

As can be seen in Fig. 1, there is a significant reduction in power absorption due to the slamming restriction. It should be mentioned that the present requirement is quite conservative. It imposes a constraint based on a certain sea state, and consequently this measure is too restrictive for the smaller waves in that sea state. It would be more efficient to implement a real time slamming restriction, than to impose a constraint that remains the same over a certain sea state. However, this would require a very reliable forecasting of the incoming waves and/ or accurate data of the current point absorber motion characteristics (buoy position with respect to free surface, buoy position relative to the wave elevation, buoy velocity and buoy acceleration). The control algorithm becomes more complicated 
and high temporary forces might be necessary to decelerate the buoy in a short time interval. In any case, the penalty to overcome slamming completely will be too high, therefore it is of importance to know to which pressure magnitudes the body is exposed when slamming occurs. The next paragraph will deal with these impact pressures and the parameters that have an influence on it.

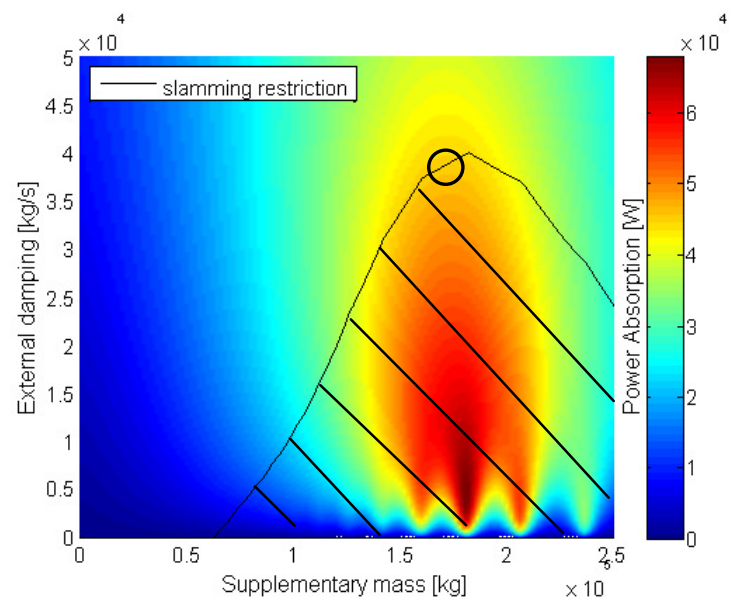

Fig. 1 Absorbed power [W] as a function of the control parameters, $B_{\text {ext }}$ and $m_{\text {sup }}$, for $H_{s}=2.25 \mathrm{~m}$ and $T_{z}=5.75 \mathrm{~s}$, with a contour line indicating the slamming restriction.

\section{ANALYTICAL INVESTIGATION}

\section{Literature overview}

One of the pioneers in slamming research is Von Karman [7], who studied water impact on rigid bodies by approximating the bodies by a growing flat plate. This work was intended to estimate the pressure on floats of hydroplanes during sea landings. Subsequent research has been carried out by Wagner [8]. He adapted the Von Karman solution by taking into account the water uprise on the body in a simplified way. Wagner mainly analyzed slamming effects on two-dimensional solid bodies. The shape of these bodies is also approximated by a growing flat plate, which implies that the Wagner method assumes small deadrise angles. Zhao and Faltinsen [9-10] presented numerically similarity solution results, based on the findings of Dobrovolskaya [11], for deadrise angles between $4^{\circ}$ and $81^{\circ}$. Based on the work of Zhao et al., Mei [12] developed an analytic solution for the water impact problem of general twodimensional bodies, where the exact body boundary conditions are fulfilled. The advantage of Wagner's approximation is the ability to use analytic expressions for the velocity potential. With the generalized Wagner method, a broader range of (local) deadrise angles can be investigated in a more accurate way. A lot of experimental work has been carried out to study the hydrodynamic impact problem and validate the analytic and numerical solutions, e.g. the work of Lin and Shieh [13], Zhao and Faltinsen [10] and Yettou [14] et al.
Most studies have focused on two-dimensional impact problems, since slamming on ships has been a major concern. However, there is a need for three-dimensional solutions, because real impact phenomena are three-dimensional. Ship designers have a growing interest in three-dimensional modelling tools, since estimates from two-dimensional solutions are not accurate enough, especially not near the bow of the ship where slamming often occurs.

Since point absorbers are considered in this paper, vertical slamming of three-dimensional objects, more specifically axisymmetric bodies are of interest. In 1945 and 1951, Shiffman and Spencer [15-16] presented solutions for the impact force on spheres and cones by approximating the bodies as a lens and an ellipsoid. One of the important contributors to axisymmetric slamming problems is Miloh [17-19], who developed analytical expressions for the slamming coefficient and slamming force. The work of Korobkin and Scolan [20-21] needs to be mentioned as well. Scolan and Korobkin [20] presented analytical solutions of the three-dimensional Wagner problem for blunt bodies with elliptic and circular contact line (an elliptic paraboloid and a cone), obtained by the inverse Wagner method and adopting the Wagner assumptions - which means that a small deadrise angle, an ideal and incompressible fluid and irrotational flow are assumed.

\section{Pressure distribution with classical Wagner method}

Despite the interesting work that has already been carried out in the field of water impact, Wagner's method is even nowadays still very valuable, since it produces analytical formulas that are easy to handle and give a very good first insight into the problem.

In this paper, the classical Wagner theory is applied to some point absorber shapes, namely hemispherical and conical buoy shapes. Since the buoys are axisymmetric, the body shape can be approximated by a growing flat disc, analogously to Wagner's flat plate approximation for two-dimensional shapes. An infinite amount of water is considered. The effect of surface tension can be neglected and the water can be assumed incompressible and the flow irrotational. Consequently, potential theory can be used to describe the flow during water entry of a rigid body. A constant entrance velocity $U$ is considered, which represents the relative velocity between the body and the water. The initial time instant $t_{0}$ is defined as the time where the body touches the calm water surface. At a time $t$, the penetration depth relative to the calm water surface $(z=0)$ equals $U t$ and the corresponding instantaneous radius at the wet section of the cone is equal to $b_{0}(t)$. However, an uprise of water is caused while the impacting body penetrates the water, as can be seen in Fig. 2.

A Von Karman method neglects this piling-up effect, whereas a Wagner method takes it into account. The instantaneous radius $b(t)$ at the intersection point between the body and the water is found by integrating the vertical velocity of the water particles at $z=0$. It should be mentioned that Fig. 2 gives a simplified presentation of the water uprise, since in 
reality a jet flow occurs which ends in a spray. This spray and the spray roots (inner domain) are not considered in this model.

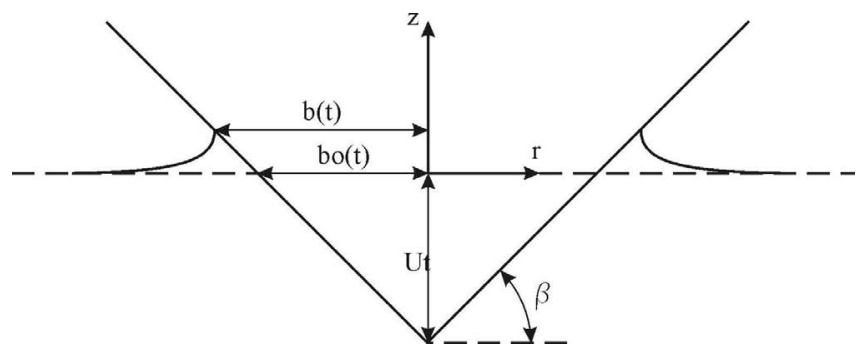

Fig. 2 Cone penetrating through originally calm water: clarification of parameters.

The generalized equation of Bernoulli is used to derive the pressure $p$ that is experienced during impact. Using the classical Wagner theory [8], the pressure on a cone shape with deadrise angle $\beta$, at a certain distance $r$ from the symmetry axis, can be described by:

$p_{\text {cone }}=\frac{1}{2} \rho U^{2}\left[1-\frac{4\left(\frac{r}{U t}\right)^{2}}{\pi^{2}\left(\frac{16}{\pi^{2} \tan ^{2} \beta}-\left(\frac{r}{U t}\right)^{2}\right)}+\frac{64}{\pi^{3} \tan ^{2} \beta \sqrt{\frac{16}{\pi^{2} \tan ^{2} \beta}-\left(\frac{r}{U t}\right)^{2}}}\right]$

The expression for the pressure is composed of three terms:

- The first term expresses the stagnation pressure.

- The second term is a consequence of the permanent flow around the disc.

- The third term accounts for the expansion of the disc. This term represents the effect of the non-stationary behaviour of the flow around the disc.

Fig. 3 shows the pressure distribution as a function of the dimensionless radius $r / U t$ for an impacting cone with different deadrise angles $\beta$. A more peaked pressure distribution and a significant pressure increase can be observed when the deadrise angle decreases, in particular for small values of $\beta$. When $\beta$ decreases from $20^{\circ}$ to $10^{\circ}$, the dimensionless pressure peak rises from 13.5 up to 53.6, which is an increase by almost a factor of 4 . Note that the pressure drops down after the peak pressure and even goes to infinity for $r$ equal to $b$, which is obviously unphysical. In order to know the correct pressure near $r=b$, an analysis near the spray roots is required. However, in order to know the total impact force, the effect of the spray can be neglected since the contribution of the pressures at the spray and spray roots is negligible.

As mentioned before, the blunt body assumption in Wagner's method implies that bodies should have small local deadrise angles. In literature, it is stated that the classical Wagner theory gives quite accurate results for wedges with deadrise angles in the range of 4 till 20 degrees [10]. Also for axisymmetric shapes, the blunt body approach will overestimate the pressures when the local deadrise angles are too high. In a second part in this paper, the analytical results will be compared with experimental measurements on cones with deadrise angles of $20^{\circ}$ and $45^{\circ}$. The theoretical results of the latter one in particular might be expected to be too conservative. When deadrise angles are smaller than 4 degrees, an air cushion is formed, which reduces the pressure on the structure.

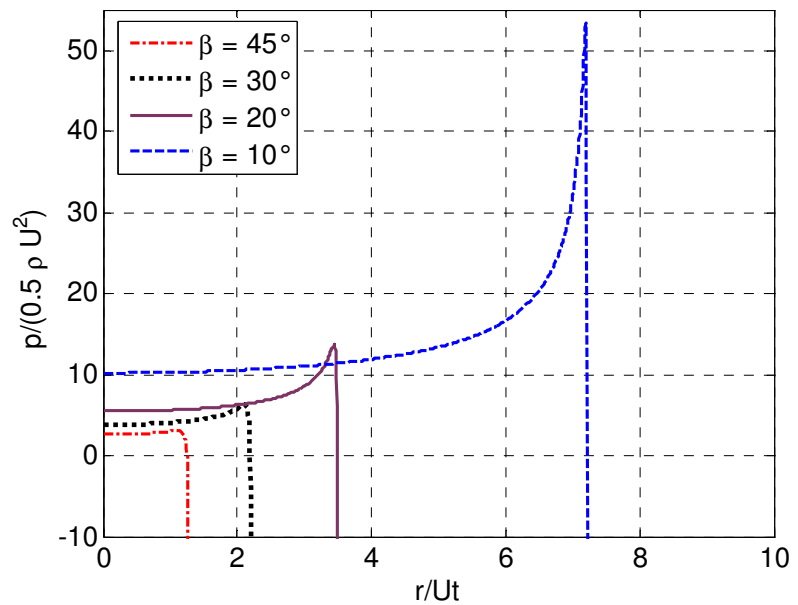

Fig. 3 Pressure distribution as a function of the dimensionless radius $(r$ divided by the instantaneous submergence $U t$ ).

In Table 1 the analytical results are compared with the results from Peseux et al. in [22], where the Wagner method has been applied numerically. The comparison is made for cones with deadrise angles $6^{\circ}, 10^{\circ}$ and $14^{\circ}$. A very good agreement is found.

Table 1 Comparison analytical results with Peseux et al. [22]
Peak pressure

(analytical)

[bar]

20.0

Cone $6^{\circ}$

Cone $\mathbf{1 0}^{\circ}$

Cone $14^{\circ}$
Peak pressure

Peseux et al. [22]

(numerical)

7.2

3.7

[bar]

19.8

7.0

3.5

In a similar way as for a cone shape, the pressure distribution has been derived for a hemispherical buoy shape. For two reasons this formula can only be applied to very small submergences. Firstly because of the disc approximation, which implies that small local deadrise angles should be considered. Secondly, the relationship between the penetration depth and instant wet radius $b$ is not as straightforward as it is for the case of a cone shape. A quadratic relation between $U t$ and $b$ has been adopted, which is only valid for small submergences $(U t / R<$ $1 / 5)$ :

$U t=\frac{b^{2}}{3 R}$ 
The pressure on an impacting hemisphere with radius $R$, at a distance $r$ from the symmetry axis, can be expressed as follows:

$p_{\text {hemisphere }}=\frac{1}{2} \rho U^{2}\left[1-\frac{4\left(\frac{r}{U t}\right)^{2}}{\pi^{2}\left(\frac{3 R}{U t}-\left(\frac{r}{U t}\right)^{2}\right)}+\frac{6}{\pi \sqrt{\frac{3 U t}{R}-\left(\frac{r}{R}\right)^{2}}}\right]$

Fig. 4 presents an example of the pressure distribution on a hemisphere with radius $R=2 \mathrm{~m}$ as a function of the distance $r$ from the symmetry axis for different penetration depths $U t$. The smaller the submergence, the smaller the local deadrise angle, which corresponds obviously with a higher pressure. Note that the pressure peak is very much localized in space, especially for small submergences.

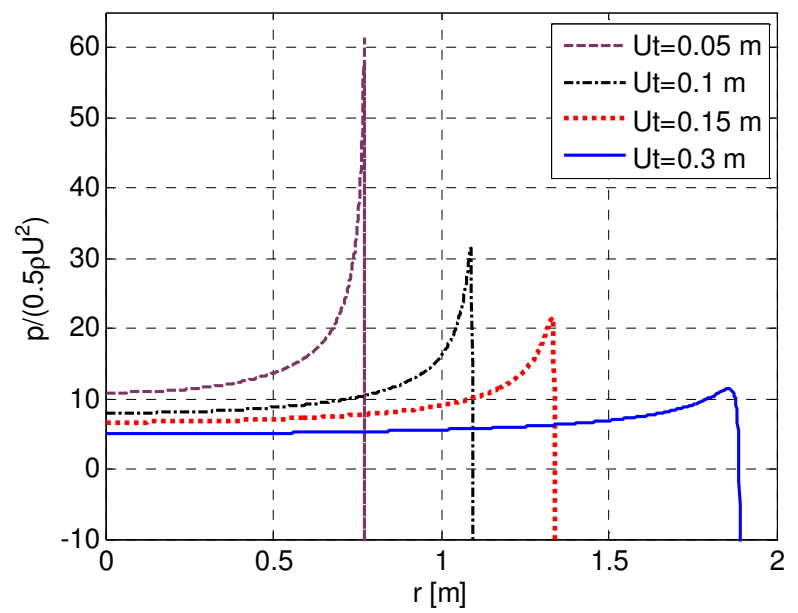

Fig. 4 Pressure distribution on a hemisphere with radius $R=2 \mathrm{~m}$, as a function of $r$, being the distance between a considered point on the hemisphere and the symmetry axis. The pressure is presented for several penetration depths.

\section{EXPERIMENTAL INVESTIGATION}

\section{Description of test setup and test models}

Free fall tests have been carried out on rigid axisymmetric bodies at Ghent University. Three rigid bodies have been tested, which are shown in Table 2: a hemisphere, a cone with deadrise angle $45^{\circ}$ and a cone with deadrise angle $20^{\circ}$. The models are made from polyurethane and have a sufficient thickness to behave as a rigid body.

A picture of the test setup is given in Fig. 5. The bodies were dropped in a water basin with dimensions ( $1 \mathrm{x} \mathrm{w} \mathrm{x} \mathrm{h)} \mathrm{of}$ $1.20 \mathrm{~m} \times 1.00 \mathrm{~m} \times 1.25 \mathrm{~m}$. Several drop heights have been evaluated up to a maximum of $4 \mathrm{~m}$, see Table 3 . Each single test was performed at least three times to have an idea of the reproducibility of the measurements. The first tests were carried out without any guiding structure, which caused too much scattering in the data. In order to make sure that the floaters fell down without experiencing small rotations, it was decided to guide the tested models along tightened steel wires.

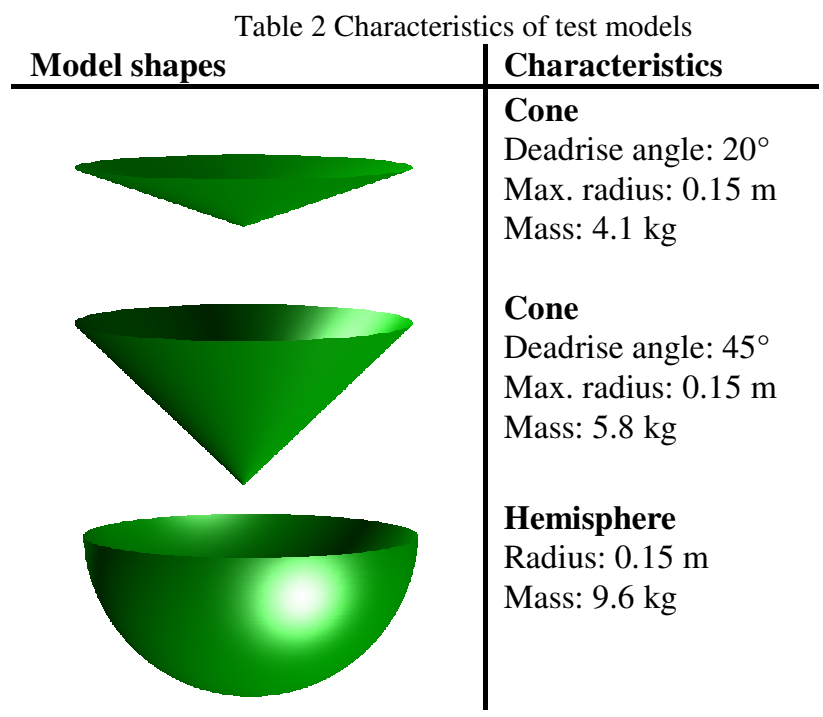

Table 3 Drop heights

\begin{tabular}{|c|c|c|c|c|c|c|}
\hline $\begin{array}{c}\text { Drop heights } \\
{[\mathrm{m}]}\end{array}$ & 0.05 & 0.10 & 0.20 & 0.30 & 0.40 & 0.50 \\
\cline { 2 - 7 } & 0.75 & 1.00 & 1.50 & 2.00 & 3.00 & 4.00 \\
\hline
\end{tabular}

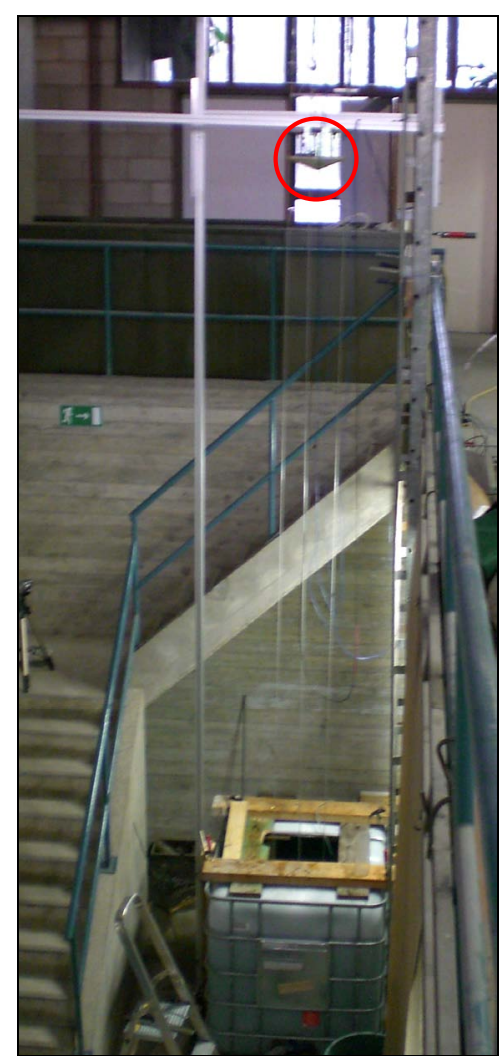

Fig. 5 Experimental test setup. The cone, at a height of $4 \mathrm{~m}$ above the water level, is encircled in red. 


\section{Instrumentation}

The pressure time history and deceleration of the body were recorded during impact. The deceleration was measured by a shock accelerometer with a range of $\pm 500 \mathrm{~g}$ and a resonance frequency of $54 \mathrm{kHz}$. Two high frequency ICP pressure sensors with a range up to $345 \mathrm{kPa}$ have been used to register the pressures. The sensors were mounted at a horizontal distance of $40 \mathrm{~mm}$ respectively $90 \mathrm{~mm}$ from the symmetry axis. Fig. 6 illustrates the sensor position for the cone with deadrise angle $20^{\circ}$.

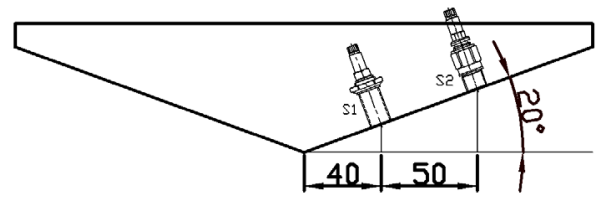

Fig. 6 Position of the pressure cells in mm: sensor 1 (S1) at $\mathrm{r}=40 \mathrm{~mm}$ and sensor $2(\mathrm{~S} 2)$ at $\mathrm{r}=90 \mathrm{~mm}$.

A sampling frequency of at least $20 \mathrm{kHz}$ was used for recording. Such high sampling frequencies are required, since the pressure peaks occur in a very small time interval (order of magnitude milliseconds). For the same reason the resonance frequency of the sensors should be high enough. The ICP sensors have a resonance frequency which is above $250 \mathrm{kHz}$ and a small membrane diameter (approximately $5 \mathrm{~mm}$ ). A small pressure cell diaphragm area is necessary, since the pressure peaks are very much localized in space as well, as can be seen in Fig. 7. Fig. 7 shows the theoretically predicted pressure distribution at $\mathrm{t}=0.002 \mathrm{~s}$ for a cone with deadrise angle $20^{\circ}$ and drop height $4 \mathrm{~m}$.

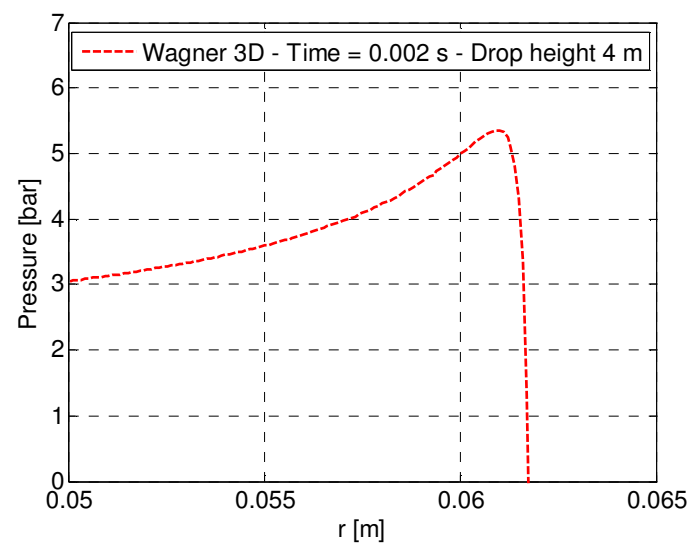

Fig. 7 Theoretical pressure distribution as a function of $r$ for a cone with deadrise angle $20^{\circ}$ and drop height: $4 \mathrm{~m}$.

In earlier investigations, sensor diameters that have been used are sometimes quite large, up to $19 \mathrm{~mm}$ in [14]. In that case the pressure peaks might have a smaller spatial extent than the sensor area. Even pressure cells with diameter $5 \mathrm{~mm}$ measure a space-averaged pressure, which is slightly different from the peak pressure. The pressure distribution is more peaked when the (local) deadrise angle is small and the impact velocity high. In [23], Faltinsen estimated that the theoretical peak pressure is at maximum $11 \%$ higher than the spaceaveraged pressure, measured by a sensor with a diameter of $4 \mathrm{~mm}$. Deviations of the same magnitude can be derived, for the pressure cells with diameter $5 \mathrm{~mm}$, based on the theoretically predicted pressure distribution. It is estimated that the measured pressure on a cone with deadrise angle $20^{\circ}$ deviates $9.7 \%$, respectively $12.5 \%$ from the peak pressure, for drop heights of $1 \mathrm{~m}$, respectively $4 \mathrm{~m}$. This should be born in mind when analyzing the results. In a similar way as above, it is expected that a pressure sensor with a diameter of $19 \mathrm{~mm}$, would underestimate the peak pressure with $30.5 \%$, respectively $34.2 \%$ for the same case of a cone with deadrise angle $20^{\circ}$.

\section{Experimental results}

Fig. 8 gives the measured pressure time history compared with the theoretically predicted pressure as a function of time. The tested body is a cone with deadrise angle $20^{\circ}$ and the drop height is $1 \mathrm{~m}$. Both the experimental and theoretical distribution show the typical shape, consisting of a very steep left side and a more gradually decreasing part on the right side of the peak pressure. The difference between the theoretical and experimental peak pressure is rather high. The possible reasons for this discrepancy are discussed later.

Piezoelectric pressure sensors are only suited to measure dynamic pressures. When pressure is applied, the quartz crystals generate a charge, which eventually leaks to zero. This is why the measured pressures drop to zero, whereas the Wagner pressures lead to the sum of the stagnation pressure and the pressure due to permanent flow.

Furthermore, it can be noticed that the time interval between the experimentally determined peak pressures is larger than between the Wagner peak pressures. This may indicate that the penetration velocity has been decreased during impact. This is confirmed by Fig. 9, where the acceleration and velocity are plotted for the same test case. The velocity drops from $4.4 \mathrm{~m} / \mathrm{s}$ to $2.6 \mathrm{~m} / \mathrm{s}$. Consequently the assumption of a constant impact velocity may lead to conservative results, especially when the bodies have a small mass.

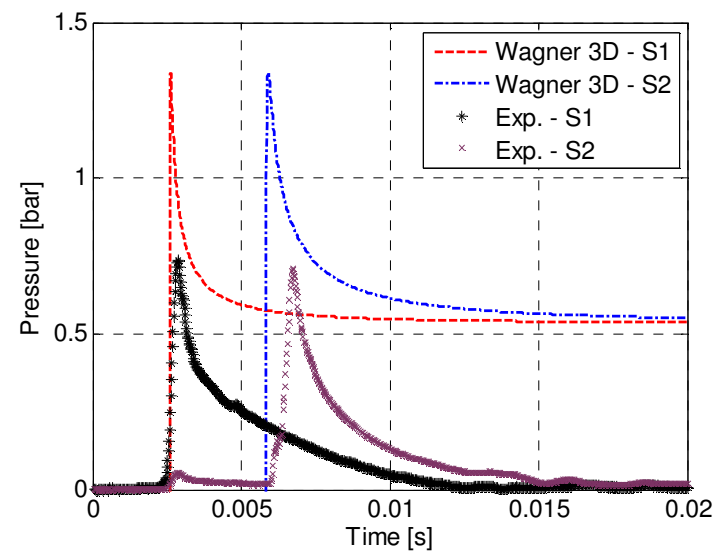

Fig. 8 Theoretical and experimental pressure time history on a cone with deadrise angle $20^{\circ}$ at positions S1 $(r=0.04 \mathrm{~m})$ and S2 $(r=$ $0.09 \mathrm{~m})$. Drop height: $1.00 \mathrm{~m}$. 


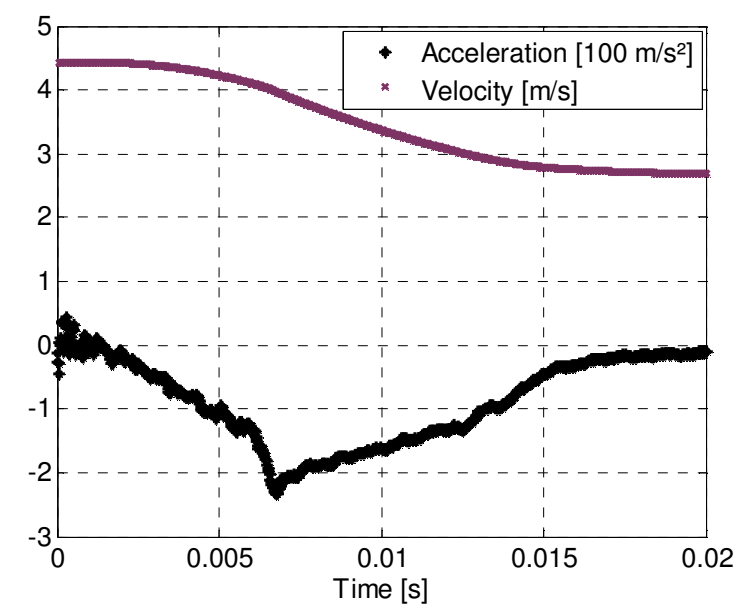

Fig. 9 Acceleration $\left[100 \mathrm{~m} / \mathrm{s}^{2}\right]$ and velocity $[\mathrm{m} / \mathrm{s}]$ as a function of time for a free falling cone (deadrise angle $20^{\circ}$ ) and drop height $1.00 \mathrm{~m}$.

In Fig. 10 the peak pressure measured on a cone with deadrise angle $20^{\circ}$ is given as a function of the drop height. The blue line shows the predicted maximum pressure obtained with the Wagner method for axisymmetric rigid bodies. It can be demonstrated that a linear correlation exists between the maximum Wagner pressure and the drop height $h$. For this reason a linear regression through the measurement points has been applied. The square of the Pearson correlation coefficient $R^{2}$ is presented, which gives an idea of the quality of the regression.

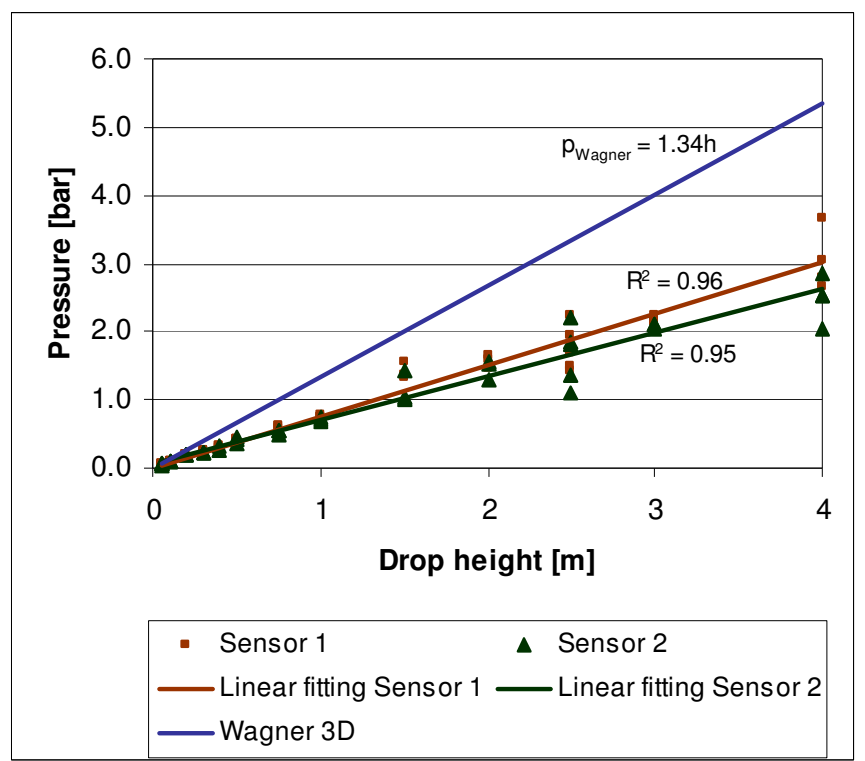

Fig. 10 Maximum pressure [bar] as function of the drop height [m] for a cone with deadrise angle $20^{\circ}$.

Fig. 11 shows the peak pressures measured on a cone with deadrise angle $45^{\circ}$ as a function of the drop height. For cone shapes, the position of the sensor is not expected to have any influence on the magnitude of the pressure. From Fig. 10 and Fig. 11, it can be seen that the pressures measured by the second sensor (at $r=0.09 \mathrm{~m}$ ) are lower than the pressures from the first sensor (at $r=0.04 \mathrm{~m}$ ). The average difference in pressure is $11 \%$, respectively $22 \%$. One of the reasons might be the velocity decrease during impact.

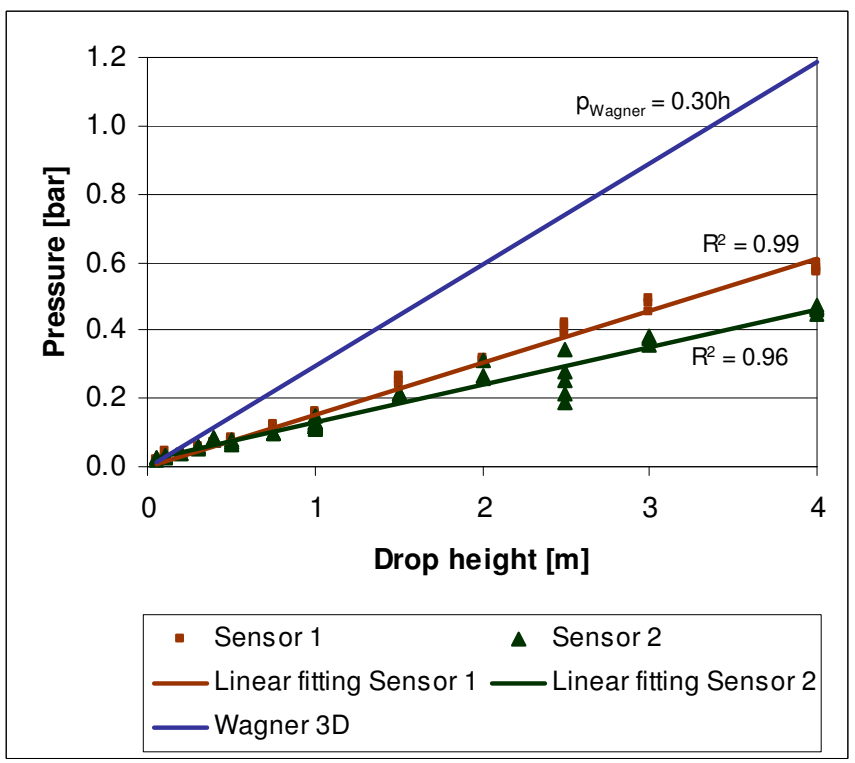

Fig. 11 Maximum pressure [bar] as a function of the drop height [m] for a cone with deadrise angle $45^{\circ}$.

Fig. 12 shows the peak pressures on a hemisphere with radius $R=0.15 \mathrm{~m}$ at two different sensor positions: $r=0.04 \mathrm{~m}$ and $r=0.09 \mathrm{~m}$. The peak pressures predicted with the Wagner method are shown by a blue, respectively red line.

From Fig. 10 - Fig. 12, it can be seen that the Wagner approach gives higher values than the measured pressures. The theoretical peak pressures on the cones with deadrise angle $20^{\circ}$ and $45^{\circ}$ are a factor of 1.9 , respectively 2.2 higher on average than the measured values. For the hemisphere the difference is a factor of 3 and 2.2 for sensor 1, respectively sensor 2 . One of the reasons for this discrepancy is the blunt body approach in Wagner's method which assumes small local deadrise angles. However, in [22], where deadrise angles between $6^{\circ}$ and $14^{\circ}$ were tested, a difference of a factor of 1.3 up to 1.9 has been found between the numerical and experimental results. This might indicate that the Wagner approach is indeed somehow conservative. Other reasons which led to an underestimation of the measured data are the pressure cell diameter, which should be preferably smaller, as mentioned before and the friction of the guiding system, which might have decreased the theoretical impact velocity. 


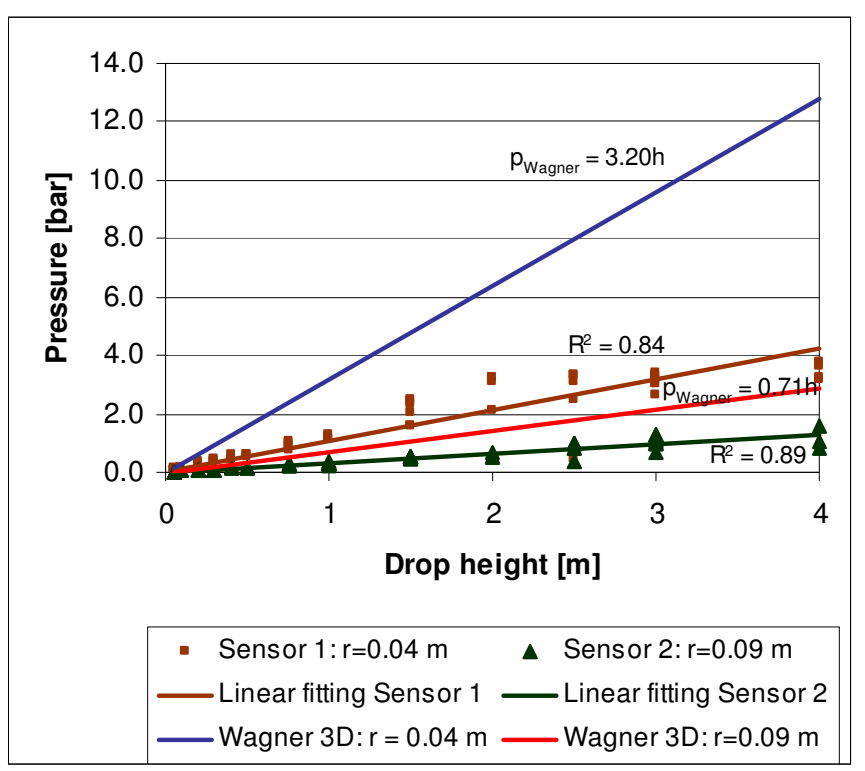

Fig. 12 Maximum pressure [bar] as a function of the drop height [m] for a hemisphere with radius $\mathrm{R}=0.15 \mathrm{~m}$. The pressure is measured at two different sensor positions, respectively at $0.04 \mathrm{~m}$ and $0.09 \mathrm{~m}$ from the symmetry axis.

\section{CONCLUSION}

In the first part, it is shown how the probability of slamming and its consequences can be reduced by controlling the point absorber motion. However, this has a quite negative influence on power absorption, especially in the case of more energetic waves and buoys with a rather small draft. A certain level of slamming will still occur in operational conditions, and also in storm conditions the point absorbers might experience vertical bottom slamming. In order to know which pressures might be expected on the buoys, an analytical investigation on the pressure distribution has been made in the second part of this paper. Analytical formulas which describe the pressure on cones and hemispheres are presented, based on the classical Wagner approach, applied to axisymmetric shapes. The results showed a very good agreement with the numerical results in [22].

Drop tests have been performed on three rigid bodies: two cones with deadrise angle $20^{\circ}$, respectively $45^{\circ}$ and a hemisphere. The pressure time history and deceleration of the body were recorded during impact. A non-negligible deceleration was measured for the cone with deadrise angle $20^{\circ}$, indicating that a constant velocity assumption might be too conservative. The theoretical pressure distribution and peak pressure are compared with the experimental results. The shape of the theoretically predicted pressure distribution is very similar to the shape of the measured distribution. However, the measured peak pressures are on average about a factor of two smaller than the theoretical peak pressures. One of the reasons is that the Wagner theory is too conservative for deadrise angles larger than $20^{\circ}$. Also the constant impact velocity approach might be too conservative for light bodies, which experience a significant deceleration. On the other hand, the measured peak pressures are expected to be too small, due to the pressure cell area and friction from the guiding system. New experiments are planned with smaller pressure sensors, a better guiding system and exact recording of the impact velocity.

\section{ACKNOWLEDGMENTS}

Research funded by Ph.D. grant of the Promotion of Innovation through Science and Technology in Flanders (IWTVlaanderen), Belgium.

The research fits into the EU project SEEWEC (Sustainable Economically Efficient Wave Energy Converter contract $n^{\circ}$ : SES6-CT2005-019969) within the $6^{\text {th }}$ Framework Programme. The additional support of the European Community is gratefully acknowledged.

The authors would like to thank Tom Versluys, Herman Van der Elst and Sam Meurez (Ghent University) for their contribution in the design and installation of the test setup and measurement infrastructure.

\section{REFERENCES}

[1] Website SEEWEC: http://www.seewec.org/

[2] Website Wave Star Energy:

http://www.wavestarenergy.com/

[3] Website Manchester Bobber:

http://www.manchesterbobber.com

[4] Vantorre, M., Banasiak, M., Verhoeven, R., 2004, "Modelling of Hydraulic Performance and Wave Energy Extraction by a Point Absorber in Heave", Applied Ocean Research 26, pp. 61-72.

[5] De Backer, G., Vantorre, M., Banasiak, R., Beels, C., De Rouck, J., 2007, "Numerical Modelling of

Wave Energy Absorption by a Floating Point Absorber System", 17th International Offshore and Polar Engineering Conference, Portugal.

[6] Alves, M., Traylor, H. and Sarmento, A., 2007, "Hydrodynamic Optimization of a Wave Energy Converter Using a Heave Motion Buoy", 7th European Wave and Tidal Energy conference, Portugal.

[7] Von Karman, T., 1929: "The Impact of Seaplane Float during Landing”, NACA, Technical Note 321, Washington.

[8] Wagner, H. (1932): "Über Stoss- und Gleitvorgänge an der Oberfläche von Flüssigkeiten”. Z. Angew Math. Mech. 12, pp. 193-215.

[9] Zhao, R., Faltinsen, O.M., 1993: "Water Entry of TwoDimensional Bodies". Journal of Fluid Mechanics 246, pp.593612.

[10] Zhao, R., Faltinsen ,O.M., Aarsnes, J., 1996: "Water Entry of Arbitrary Two-Dimensional Sections With and Without Flow Separation". ONR, Norway.

[11] Dobrovolskaya, Z.N., 1969: "On Some Problems of Similarity Flow of Fluid With a Free Surface", Journal of Fluid Mechanics 36(4). 
[12] Mei, X., Liu, Y., Yue, D.K.P., 1999: "On the Water Impact of General Two-Dimensional Sections", Applied Ocean Research 21, pp.1-15.

[13] Lin, M.-C., Shieh, L.-D., 1997: "Flow Visualization and Pressure Characteristics of a Cylinder for Water Impact, Applied Ocean Research 19, pp. 101-112.

[14] Yettou, E.-M., Desrochers, A., Champoux, Y., 2006: "Experimental Study on the Water Impact of a Symmetrical Wedge", Fluid Dynamics Research 38, pp. 47-66.

[15] Schiffman, M., Spencer, D.C., 1945: "The Force of Impact on a Sphere Striking a Water Surface", Appl. Math. Panel Rep. 42 IR AMG-NYU No. 105.

[16] Schiffman M., Spencer D.C., 1951: "The Force of Impact on a Cone Striking a Water Surface", Comm. Pure Appl. Math 4 pp. 371-417.

[17] Miloh, T., 1981: "Wave Slam on a Sphere Penetrating a Free Surface, Journal of Engineering Mathematics, 15 (3), pp. 221-240.

[18] Miloh, T., 1991: "On the Oblique Water-Entry Problem of a Rigid Sphere", Journal of Engineering Mathematics, 25, pp. 77-92.

[19] Miloh, T., 1991: "On the Initial-Stage Slamming of a Rigid Sphere in a Vertical Water Entry", Applied Ocean Research, 13 (1), pp.34-48.

[20] Scolan, Y.M., Korobkin, A.A., 2001: "Three-Dimensional Theory of Water Impact. Part1: Inverse Wagner problem", Journal of Fluid Mechanics, 440, pp. 293-326.

[21] Korobkin, A.A., Scolan, Y.-M., 2006: "Three Dimensional Theory of Water Impact. Part 2. Linearized Wagner problem".

[22] Peseux, B., Gornet, L., Donguy, B., 2005: "Hydrodynamic impact : Numerical and experimental investigations", Journal of Fluids and Structures 21, pp. 277-303.

[23] Faltinsen, O.M., Chezhian, M., 2005: "A generalized Wagner Method for Three-Dimensional Slamming", Journal of Ship Research, 24 (4), pp. 279-287. 